\title{
Traffic Derived Particulate Matter Triggers Ocular Surface Inflammation in Murine Model
}

\author{
Hyun Soo Lee ${ }^{1}$, Ji Young Kwon ${ }^{1}$, Sehyun Han ${ }^{2}$, Ki-Jun Jeon ${ }^{2}$ \\ ${ }^{1}$ Department of Ophthalmology, St. Paul's Hospital, College of Medicine, The Catholic University of Korea \\ Seoul, Republic of Korea \\ ${ }^{2}$ Department of Environmental Engineering, Inha University \\ Incheon 22212, South Korea \\ cotranmd@gmail.com
}

\section{Extended Abstract}

Objective: According to the recent industrialization and urbanization, air pollution has been identified as a major risk factor for respiratory, cardiovascular, and skin diseases and increased morbidity and mortality worldwide in these days [1, 3]. Polluted air causes subclinical inflammation and dryness on the ocular surface.4-7 Previous studies have focused on air pollution from fine particles (PM2.5; particulate matter less than $2.5 \mathrm{~mm}$ in aerodynamic diameter) [3,4,5]. The aim of this study is to evaluate the mechanism of environmental eye disease caused by the exposure to airborne PM2.5 using animal experiment models.

Methods: 7-8 week old C57BL/6 mice were exposed with topical application of PM2.5, PM10, and saline vehicle to induce experimental environmental eye disease model. Corneal fluorescein staining and the number of corneal CD11b+cells were assessed in the different groups. Expression of IL-1 $\beta$, IL-6, tumor necrosis factor (TNF)- $\alpha$, IL-17A, and MUC5AC, were evaluated by real-time PCR in the corneas at day 5 or 14. TUNEL assay was used to evaluate apoptosis of corneal epithelial cells.

Results: Treatment with PM2.5 and PM10 showed a significant increase in corneal fluorescein staining compared with the vehicle, but not statistically different in between PM2.5 and PM10 group. A significant increase in the number of CD11b+ cells was observed in PM2.5 and PM10 treated eyes, compared with the vehicle in the central corneas. Exposure with PM2.5 and PM10 was associated with a significant increase in the corneal expression of IL-1 $\beta$, IL-6, IL-17, and TNF compared to the vehicle, and increased maturation of APCs in drainage LNs. In addition, cellular toxicity to the corneal epithelium was observed with PM2.5 and PM10.

Conclusions: Exposure to traffic derived PM induced ocular surface damage and inflammation, which induce the maturation of APCs in drainage cervical LNs.

Acknowledgement: This study was funded by the Korea Ministry of Environment (MOE), as the Environmental Health Action Program (2016001360005).

\section{References}

[1] R. D. Brook, S. Rajagopalan, C. A. Pope III, et al, "Particulate matter air pollution and cardiovascular disease: an update to the scientific statement from the American Heart Association," Circulation, vol. 121, pp. 2331-2378, 2010.

[2] R. Beelen, O. Raaschou-Nielsen, M. Stafoggia, et al, "Effects of long-term exposure to air pollution on natural-cause mortality: an analysis of 22 European cohorts within the multicentre ESCAPE project," Lancet, vol. 383, pp. 785795, 2014.

[3] P. Novaes, P. H. Saldiva, M. Matsuda, et al, "The effects of chronic exposure to traffic derived air pollution on the ocular surface," Environ Res., vol. 110, pp. 372-374, 2010.

[4] F. K. Malerbi, L. C. Martins, P. H. Saldiva, A. L. Braga, "Ambient levels of air pollution induce clinical worsening of blepharitis," Environ Res., vol. 112, pp. 199-203, 2012.

[5] A. Galor, N. Kumar, W. Feuer, D. J. Lee, "Environmental factors affect the risk of dry eye syndrome in a United States veteran population," Ophthalmology, vol. 121, no. 972-973, 2014. 